\title{
Donepezil overdose - trending levels and symptoms
}

\author{
Allan R. Mottram ${ }^{1}$, Elise ter Haar ${ }^{2}$ \\ 1. Section of Emergency Medicine, University of Wisconsin, Madison WI, United States. 2. Section of Emergency Medicine, \\ University of Wisconsin, Madison WI, United States.
}

Correspondence: Allan R. Mottram. Address: Section of Emergency Medicine, University of Wisconsin, Madison WI, United States. Email: armottram@medicine.wisc.edu

Received: March 5, 2014

DOI : $10.5430 /$ crim.v1n2p80
Accepted: April 2, $2014 \quad$ Online Published: April 10, 2014

URL: http://dx.doi.org/10.5430/crim.v1n2p80

\section{Abstract}

Donepezil (Aricept) is a reversible acetylcholinesterase inhibitor used in the treatment of dementia and cognitive impairment. Side effects of the drug include nausea, vomiting, diarrhea, muscle cramps, dizziness, fatigue and anorexia. Cholinergic symptoms may occur with overdose and may be severe. We report a case of donepezil toxicity with significant bradycardia during which serial donepezil levels were measured, and are correlated with the patients’ symptoms.

\section{Keywords}

Donepezil, Cholinergic, Toxicity, Bradycardia

\section{I ntroduction}

Donepezil (Aricept) is a reversible acetylcholinesterase inhibitor used in the treatment of mild to moderate dementia, and has been used in the treatment of mild cognitive impairment although this practice is not well supported ${ }^{[1-4]}$. Therapeutic levels are attained with doses of 5-10 mg/day, and peak plasma concentrations are reached 3-4 hours after oral administration. Its half life of elimination is 70 hours. Side effects of the drug include nausea, vomiting, diarrhea, muscle cramps, dizziness, fatigue and anorexia. Symptomatic bradycardia, respiratory insufficiency, syncope and complete AV block have also been reported ${ }^{[5-9]}$. We report a case of donepezil toxicity during which serial donepezil levels were measured, and are correlated with the patients symptoms.

\section{Case report}

A 53 year old otherwise healthy male with a history of memory loss was placed on donepezil by his primary care physician six months prior to his emergency department visit. The emergency department visit was for an acute accidental ingestion of up to twenty 10mg donepezil tablets, a dose that is 20-40 times the usual dose of donepezil. The patient reported ingesting the pills due to mistaking the bottle for his multivitamins, however the reliability of this history was questionable. He presented to the emergency department via ambulance approximately thirty to sixty minutes after the ingestion. It was reported that he immediately "felt sick", sleepy and vomited twice. He was confused, somnolent, diaphoretic and bradycardic with heart rate in mid 30's to 50's in emergency department. An electrocardiogram demonstrated sinus bradycardia. 1mg intravenous atropine was administered due to the patients confusion and hemodynamic instability. A chest xray was obtained and demonstrated mild pulmonary congestion. A basic metabolic 
panel and complete blood count were noted to be within the normal reference ranges. He was admitted to the medical intensive care unit for monitoring and supportive care.

The patient's vital signs and cholinergic symptoms (nausea, vomiting, diarrhea and skin parameters) were recorded every 2 hours for 16 hours and then every four hours for the rest of his hospitalization. The patient was somnolent, nauseous and feeling cold (room temperature 80 degrees F) for first 8 hours. Over the next 8-16 hours, the patient became more alert and conversant, however he did have an episode of recurrent nausea and diaphoresis at 16 hours which was associated with food intake.

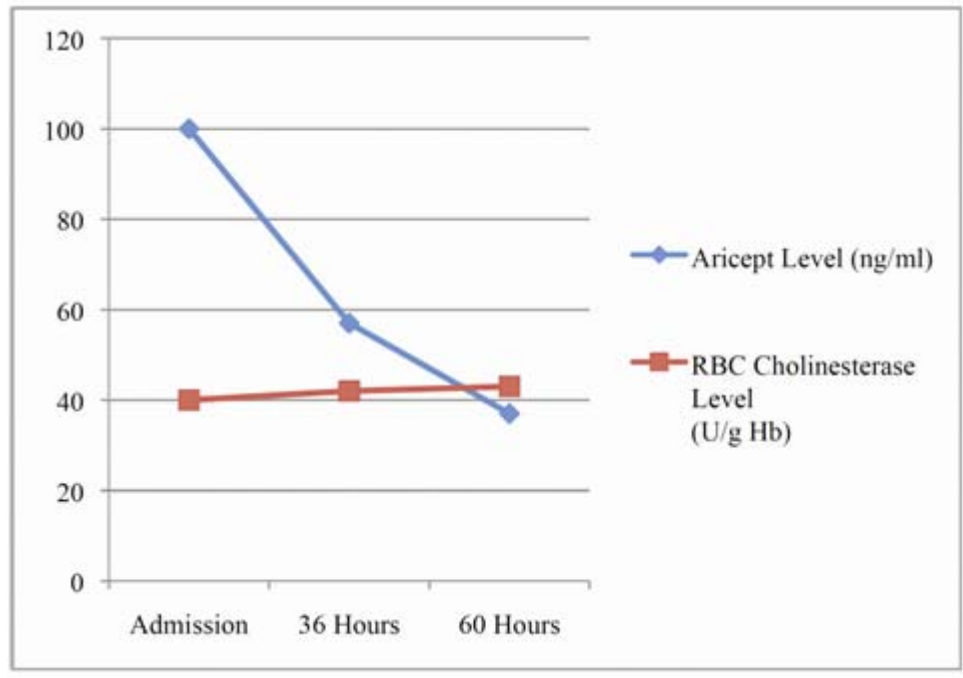

Figure 1. Trends of Donepezil and RBC Cholinesterase

Serum levels of donepezil and red blood cell cholinesterase levels were drawn in the emergency department and trended during his hospital stay. The initial donepezil level was $100 \mathrm{ng} / \mathrm{ml}$ and decreased over three days to $57 \mathrm{ng} / \mathrm{ml}$ at 36 hours, and 37mg/ml at 60 hours. The patients heart rate at these time points was 30-50 bpm (initial), $57 \mathrm{bpm}$ (36 hours), and 64 bpm (60 hours). This bradycardia was not associated with hypotension. The trend in decreasing donepezil levels paralleled improvement in the patients other symptoms, with the cholinergic symptoms resolving over first 16 hours. The patient was discharged on the third hospital day with a heart rate of 64 and feeling much better. It was recommended that he stop using donepezil upon discharge.

\section{Discussion}

Donepezil was used in this case for treatment of mild cognitive impairment, though it should be noted that there is a lack of evidence to support this practice. In patients with cognitive impairment associated with Alzheimer's disease, however, there is associated loss of cholinergic neurons within the brain and this cholinesterase inhibitor is thought to improve the function of remaining cholinergic neurons. In the case of our patient, he had been prescribed the medication and had been compliant with it for 6 months prior to the ingestion, so it is likely that he had a therapeutic level prior to the accidental ingestion. The initial donepezil level was $100 \mathrm{ng} / \mathrm{ml}$, which is significantly higher than what has been reported previously in a case of donepezil toxicity, with the prior reported level being $54.6 \mathrm{ng} / \mathrm{ml}$. In this case, the patients donepezil level decreased over the following three days to $37 \mathrm{ng} / \mathrm{ml}$, which correlated with an improvement, then resolution, of symptoms on hospital day three. The estimated half life for elimination was just under 42 hours. The RBC anticholinesterase level was within normal range during entire hospitalization, but increased from 40 to $43 \mathrm{U} / \mathrm{g}$ hemoglobin over the hospitalization. 


\section{Conclusion}

We report a case of donepezil overdose with serial donepezil levels. Our case report differs from prior cases in that the reported levels are higher, and the elimination half life appears shorter ${ }^{[5]}$. While this does not per se make our report unique, this variability in case reports is important as it speaks to the unpredictable nature of pharmacokinetics when considering supratherapeutic levels of drugs. Further, while we aim to provide a quantitative measure of this toxicity, one should, in general, treat the patient and not the "drug level".

This patient experienced cholinergic symptoms with nausea, vomiting, confusion, somnolence, diaphoresis, and bradycardia. These symptoms were most prominent at donepezil levels of $100 \mathrm{ng} / \mathrm{ml}$, and resolved gradually over three days. The patient was asymptomatic at a donepezil level of $37 \mathrm{ng} / \mathrm{ml}$. The patients symptomatic bradycardia occurred promptly after his ingestion, and responded well to intravenous atropine; he never demonstrated hypotension. He was admitted to the intensive care unit but did not require any further intervention during the remainder of his hospitalization, and was discharged in good condition.

This case illustrates that the effects of a donepezil overdose can be immediate and decrease over time with the elimination of the drug. Further, this case report supports the use of standard interventions such as atropine and good supportive care with adequate observation for mild to moderate cholinergic symptoms from donepezil toxcity.

\section{References}

[1] Román GC, Rogers SJ. Expert Opin Pharmacother. Donepezil: a clinical review of current and emerging indications. 2004 Jan; 5(1): 161-80.

[2] Farlow MR. Current Alzheimer Research. Treatment of Mild Cognitive Impairment (MCI). 2009; 6: 362-367.

[3] Petersen RC et al. for the Alzheimer’s Disease Cooperative Study Group. NEJM. Vitamin E and Donepezil for the Treatment of Mild Cognitive Impairment. 2005 June; 352 (23).

[4] Doody RS et al. American Journal of Alzheimer's Disease \& Other Dementias. Safety and Tolerability of Donepezil in Mild Cognitive Impairment: Open-Label Extension Study. 25(2): 155-159.

[5] Yano H, Fukuhara Y, Wada K, Kowa H, Nakashima K. Rinsho Shinkeigaku. A case of acute cholinergic adverse effects induced by donepezil overdose: a follow-up of clinical course and plasma concentration of donepezil. 2003 Aug; 43(8): 482-6. Japanese.

[6] Shepherd G, Klein-Schwartz W, Edwards R. Ann Pharmacother. Donepezil overdose: a tenfold dosing error. 1999 Jul-Aug; 33(7-8): 812-5.

[7] Calvo-Romero JM, Ramos-Salado JL. Rev Neurol. Symptomatic sinus bradycardia associated with donepezil. 1999 Jun 1-15; 28(11): 1070-2. Spanish.

[8] Tanaka A, Koga S, Hiramatsu Y. Donepezil-Induced Adverse Side Effects of Cardiac Rhythm: 2 Cases Report of Atrioventricular Block and Torsade de Pointes. Inter Med. 2009; 48: 1219-1223. http://dx.doi.org/10.2169/internalmedicine.48.2181

[9] Suleyman T, Tevfik P, Abdulkadir G, Ozlem S. Complete atrioventricular block and ventricular tachyarrhythmia associated with donepezil. Emerg Med J. 2006; 23: 641-642. http://dx.doi.org/10.1136/emj.2006.036251 